\title{
REFLECTANCE AND PHOTOEMISSION FROM Ag IN LIQUID NH
}

\author{
H.-T. CHOU and J.C. THOMPSON
}

Department of Physics, University of Texas at Austin, Austin, TX 78712, U.S.A.

\begin{abstract}
We have made simultaneous observations of reflectance and photoyield from $\mathrm{Ag}$ films in liquid $\mathrm{NH}_{3}$. Photoyield is at the angle for surface plasmon excitation. The yield varies linearly with laser intensity. The results can be interpreted in terms of the classical Fresnel equations. The effective dielectric constant for $\mathrm{NH}_{3}$ is 1.95 instead of 1.75 .
\end{abstract}

\section{1.-Introduction}

Photoinjection thresholds in insulating fluids, such as $\mathrm{CH}_{3}$ or $\mathrm{Ar}$, provide a measure of the location of the conduction band in the material and of photoelectron mobilities /1-3/. There are two effects when a polar fluid is placed adjacent to a photocathode $14,5 /$. First, a potential shifts all electronic levels in the fluid relative to those in the electrode $15 /$. The second effect is that the photo-threshold is reduced, as electrons are injected into electronic states of the fluid /1/ rather than the vacuum. Bennett, et al. /6/ have docymented these effects for $\mathrm{Ag}$ electrodes in liquid $\mathrm{NH}_{3}$. Disagreement exists as to the photoyield dependence on cell potential, and as to any effect of the dipole field on measurement of the position of the conduction band 14-12/.

Watanabe and Gerischer $/ 10 /$ attributed the tail on the photothreshold curve to oxide on their Au electrode. In an oxide, charge is pulled from the metal to the oxygen In contrast, with Cs, charge is shifted from the atom toward the metal. Cs is well known to lower threshoids; therefore, one expects oxides to raise the threshold. Mee and coworkers $/ 14 /$ have demonstrated an increase in threshold on $\mathrm{Ag}$ with the addition of $\mathrm{O}_{2}$ and $\mathrm{S}_{2}$. We prefer a tail on the density of states.

The position of the surface plasmon polariton, SPP, resonance, $\theta_{S}$, is shifted by the dielectric constant of a semi-infinite fluid, such as $\mathrm{NH}_{3}$, placed in contact with the Ag:

$$
\mathrm{n}^{2} \sin { }^{2} \theta_{S}=\left[\varepsilon_{1}(\mathrm{Ag}) \varepsilon_{1}\left(\mathrm{NH}_{3}\right)\right] /\left[\varepsilon_{1}(\mathrm{Ag})+\varepsilon_{1}\left(\mathrm{NH}_{3}\right)\right]
$$

where $\varepsilon_{1}(\mathrm{Ag})$ and $\varepsilon_{1}\left(\mathrm{NH}_{3}\right)$ are the real parts of the $\mathrm{Ag}$ and $\mathrm{NH}_{3}$ dielectric constants, respectively, and $n$ is the prism index of refraction $/ 14 /$. The critical angle also depends on $\varepsilon_{1}\left(\mathrm{NH}_{3}\right)$ and $\mathrm{n}$. Measurements /15/ on $\mathrm{Ag}$ films in $\mathrm{H}_{2} \mathrm{O}$ show the electrode potential changes the SPP resonance energy, as well as the 
disipersion curve near the potential of zero charge. Potential extracts charge from the electrode and causes a shift in the metal surface dielectric constant. We have measured photocurrents in liquid $\mathrm{NH}_{3}$ in the attenuated total reflection, ATR, configuration. In contrast to the gas case $/ 16 /$, we find the photoyield to depend only on the first power of the laser intensity. We have looked at the trends of photoyield with bias 16-8,10\% Separately, we have also examined the dielectric constants derived from the reflectance data and inferred from them the nature of the interface between the metal and fluid $15,15 /$ The photoyield may be calculated from the measured dielectric constants.

\section{2.-Experimental}

The apparatus is identical to that we have used in our study of photoemission induced by second harmonic photons in the ATR configuration $116 /$ Light is reflected by 3 . Ag film denssited onto the flat face of a hemicylindrical prism. The prism is rotated to vary the angle of incidence. $\mathrm{NH}_{3}$ is condensed at $216 \mathrm{~K}$ into a quartz vessel surrounding the prism and electrodes. The anode and reference electrodes are $\mathrm{Ag}$ and rotate so that they are always in the same position with respect to the photocathode. Bias is provided to the collector through a modified Ortec 142PC amplifier $16 \%$. All of our surfaces are exposed to air prior to pumping and immersion in $\mathrm{NH}_{3}$.

Photon energies from 1.8 to $2.6 \mathrm{eV}$ are provided by a Molectron pulsed dye laser. Powers up to $2.8 \times 10^{7} \mathrm{~W} / \mathrm{cm}^{2}$ are avallable. High laser intensity can damage the metal films and change the ATR. Undamaged films were used for the data here.

Signals go to a Macintosh II personal computer that uses LabView software and hardware. The photoemission and reflectance are recorded simultaneously as furictions of the angle of incidence with a resolution of 19 points per degree. The angular scan rate is 0.0534 degree per second.

The maximum ratio of collected photoelectrons to incident photons is $1.4 \times 10^{-7}$.

\section{3.-Results and Discussion}

Runs were made with $\mathrm{Ag}$ films of different thicknesses. Since the vacuum chamber could not be baked, there were differences in the residual impurities, particularly $\mathrm{H}_{2} \mathrm{O}$. From previous work $/ 6 /$ we know that $\mathrm{H}_{2} \mathrm{O}$ causes changes in the photoyield. There are run-to-run variations in our present results, as well.

Figure 1 shows typical data. There is no response with S-polarization. The present results are similar to those with only a monolayer of $\mathrm{NH}_{3} / 16 /$. Note that the photoyield peak is wider than the reflectance minimum with a tall to large angles. The positions of the reflectance minima, $\phi_{\mathrm{m}}$, and photoyield maxima, $\phi \mathrm{M}$, respectively, are not coincident.

As in Eq. (1), $\varepsilon_{1}\left(\mathrm{NH}_{3}\right)$ determines the dispersion curve of the surface plasmon resonance 114/. Since the rate of change of the resonant angle with either of the two dielectric constants is proportional to the other, the effect of the $\mathrm{NH}_{3}$ is greater than the metal. The shift of the SPP resonance from its vacuum value is consistent with an $\varepsilon_{1}\left(\mathrm{NH}_{3}\right)$ of 1.91 rather than of 1.76 . The same large $\varepsilon_{1}\left(\mathrm{NH}_{3}\right)$ 
is derived from the observed value of the critical angle. These observations suggest that the perturbed $\varepsilon_{1}\left(\mathrm{NH}_{3}\right)$ must apply for 10 's of $\mathrm{nm}$ into the fluid, not just at the electrode.

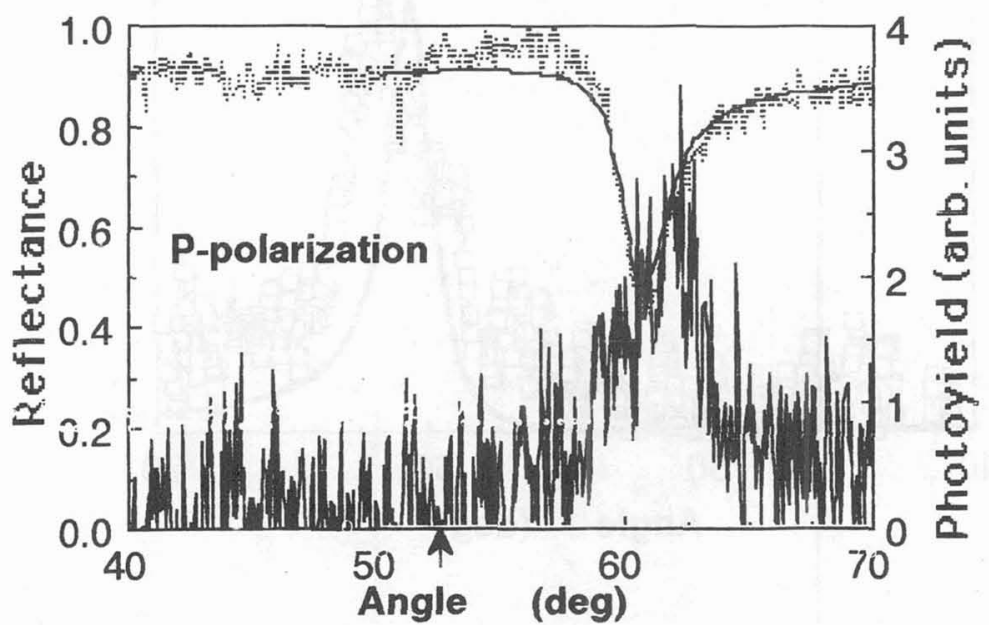

Fig. 1-Reflectance (dashed line) and photoyield (solid line) data vs. angle of incidence for P-polarized light. The photon energy was 2.363 $\mathrm{eV}$ and the potential $-1.29 \mathrm{~V}$ with respect to a $\mathrm{Ag}$ reference electrode. The arrow marks the critical angle. The reflectance curve was calculated using values of the $\mathrm{Ag}$ dielectric constants from Ref. 15.

The solvent molecules near the electrode do not behave as do those in the bulk fluid. There is no added electrolyte, so that the Debye screening length is long. Double layers do not obviously lead to an increased $\varepsilon_{1}\left(\mathrm{NH}_{3}\right)$. The $\mathrm{NH}_{3}$ lone-pair is dorated to the bonding process with $\mathrm{Ag}$, leaving the molecule more polarizable $117 /$, but only at the interface. A second problem is that the data require values of $E_{2}\left(\mathrm{NH}_{3}\right)$ near 0.02 . The absorption constant of bulk $\mathrm{NH}_{3}$ is much smaller. Such values of $\varepsilon_{2}\left(\mathrm{NH}_{3}\right)$ are understandable if there is an absorber in the liquid. Solvated electrons show a broad optical absorption peaked near $0.8 \mathrm{eV}$, and extending up above $2 \mathrm{eV}$. Ample evidence for solvated electron formation from photoinjected electrons in liquid $\mathrm{NH}_{3}$ has been found in the past /6,9/. Any modification of optical constants by a surface film would have to be potential indespendent as the values of $\varepsilon_{1}\left(\mathrm{NH}_{3}\right)$ and $\varepsilon_{2}\left(\mathrm{NH}_{3}\right)$ are independent of potential.

Dielectric constants $\varepsilon_{1}(\mathrm{Ag})$ and $\varepsilon_{2}(\mathrm{Ag})$ for $\mathrm{Ag}$ are close to literature values /14/. In the present work, there is a persistent shift, $\Delta=\phi_{M^{-}} \phi_{m}$, of about $+1 \mathrm{deg}$. Previous authors /18-20/ have found agreement between calculations based on the Fresnel relations $/ 14]$ and photoemission observed in vacuum with an adsorbate. The optical constants and film thicknesses for the reflectance are not the same as for the photoyield. Only $\varepsilon_{2}\left(\mathrm{NH}_{3}\right)$ can affect $\Delta$. Yet, fitting the photoyield requires $\varepsilon_{2}\left(\mathrm{NH}_{3}\right)$ so large $(0.05)$ that the computed reflectance minimum is half that observed. Neither set of $\mathrm{NH}_{3}$ parameters is consistent with the literature. Figure 2 shows an example of the jioblem. The reflectance data 


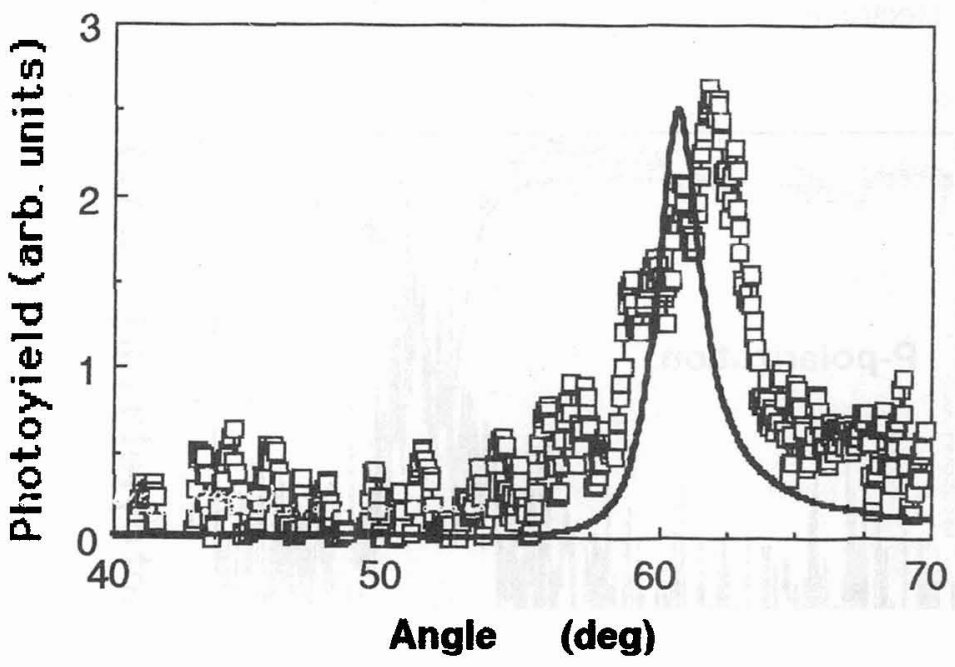

Fig. 2-Example of the quality of fit for the photoyield. The offset and high angle tail are typical.

is similar to Fig. 1, yet there is a shift between the measured $\phi_{M}$ and that calculated using the same set of parameters as in the reflectance.

In a few cases, measurements with S-polarized light showed a photoyield at angles above $\phi_{\mathrm{m}}$ but not at $\phi_{\mathrm{m}}$. It is possible, then, that a volume contribution 119 / to the measured photoyield broadens and shifts curves such as Fig. 2 . The origin of a volume contribution is obscure. Coupling of S-polarized light to produce photoemission is enhanced on a rough surface /14/. Our photocathodes become rough during the course of an experiment.

In summary, the $\mathrm{Ag}$ dielectric constants seem normal yet the values required for $\mathrm{NH}_{3}$ are not. Neither set of parameters is successful in explaining the difference between $\phi_{M}$ and $\phi_{\mathrm{m}}$. We must look to solvent structure or surface electronic states or to solvated electrons in the bulk fiuid for an explanation.

As expected, the reflectance and photoemission curves also depend on reference potential. The broad, asymmetric shape of the photoyield curve makes definition of the peak position ambiguous, as in Fig. 2. There is much more scatter in $\phi M$ than in $\phi_{m}$. The value of $\phi_{M}$ shifts by less than a degree over the entire bias range; $\phi_{m}$ doesn't shift at all. (Measurements /15/ in $\mathrm{H}_{2} \mathrm{O}$ show shifts equivalent to a small fraction of a degree that would be invisible to us.)

The photothreshold is shifted with an applied potential at fixed photon energy $|5,6|$. In Fig. 3 the square root $|6|$ of the peak yield vs. potential (relative to a $\mathrm{Ag}$ reference electrode) is shown for photon energies of 2.100 and $2.362 \mathrm{eV}$. The threshold shift is near the $0.26 \mathrm{eV}$ difference in photon energies. The figure also shows the "two slope" behavior found for conventional photoinjection into polar fluids $16-12 /$. The resonance angles and the magnitude of the yield, as in Fig. 3 , 
do not show the same changes with bias. The shift in photothreshold has been attributed $|6|$ to the liquid density of electronic states. The present results suggest that there is no real shift in the interfacial dielectric constants just as would be expected if the "two slope" behavior were a final state effect.

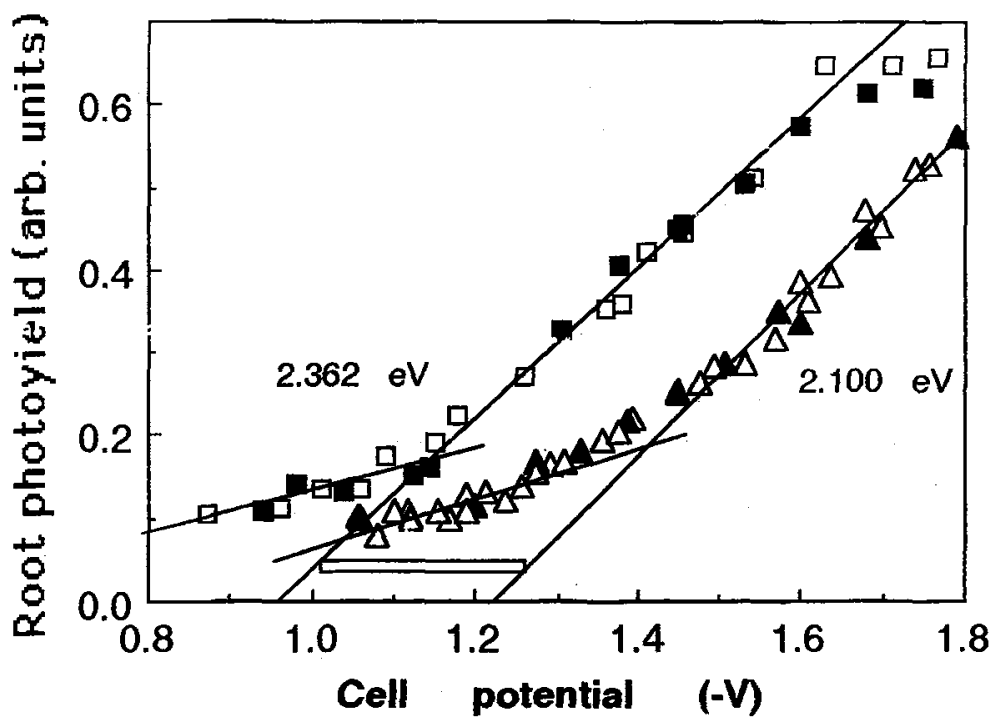

Fig. 3-Square root of peak photoyleld vs. potential for two photon energies. The root is chosen for ordinate as that is the prediction of the Fowler model, see Ref. 6 . The small box near $1.1 \mathrm{~V}$ represents the potential shift anticipated from the difference in photon energies.

The power of ATR lies in its surface specificity. Yet even more surface sersitivity is provided by photoemission, in part because of the limited escape length and in part because of changes in the surface barrier. In the electrolyte environment, the double layer modifies the surface barrier through a large electric field. This field is smaller than with a molecular monolayer. Because of solvent-solvent interactions in the fluid the molecular alignment is far less perject and we do not see second harmonic generation /16/. Variations in surlace morphology of the $\mathrm{Ag}$ films and in electrolyte purity combine to introduce a wide variability in our observations. Nevertheless, several points persist from run to run and appear reliable. The $\mathrm{Ag}$ surface is close to normal, yet these measurements reveal a far more complex surface environment than did the previous direct photoemission results. While the previous measurements could be related to the existence of shallow electron traps in the equilibrium fluid, these data require that the fluid in the neighborhood of the photocathode be far from bulk equilibrium. The present results suggest that the concentration of absorbers near the electrode is increased and the local dielectric constant is modified. Whether the perturbed values of the $\mathrm{NH}_{3}$ dielectric constant can be understood as a by-product of the double layer, or whether a more specific 
solvent/electrode interaction is required cannot be yet determined. In either event, potential has little effect on the parameters of the interface.

We see different dielectric constants necessary for the interpretation of the ATR and for the photoemission dispersion curves and line shapes. We attribute this to the different parts of fluid and metal sampled by light and by photoelectrons. We do not understand the photoyield induced by S-polarized light in some cases.

Acknowledgements

This work has been supported by the R. A. Welch Foundation and by the Texas Acvanced Research Program. T. H. Koschmieder wrote most of the software.

References

/1/R. M. Stratt, Ann. Rov. Phys. Chem. 41 (1990) 175.

/2/A. A. Holroyd and W. F. Schmidt, Ann. Rev. Phys. Chem. 40 (1989) 439.

/3/G. R. Freeman, in Kinetics of Nonhomogeneous Processes, G. R. Freeman, ed. (Wiley, New York, 1987) Chapt 2., p.19.

14/J. $K$ Sass and $H$. Gerischer, in Photoemission and the Electronic Properties of Surfaces, B. Feuerbacher, B. Fitton, and R. F. Willis, eds. (Wiley, New York, 1978), p. 469.

/5/D. M. Kolb and C. Franke, Appl. Phys. A 49 (1989) 379.

16/G. T. Bennett, R. B. Coffman, and J. C. Thompson, J. Chem. Phys. 87 (1987) 7242.

I7!A. M. Brodsky and Yu. Ya. Gurevich, Zh. Eksp. Teor. Fiz. 54 (1968) 213.

/8/K. Itaya, R. E. Malpas, and A. J. Bard, Chem. Phys. Letters 63 (1979) 411; J. Am. Chiem. Soc. 101 (1979) 2535.

/9/C. E. Krohn, P. R. Antoniewicz, and J. C. Thompson, Surf. Sci. 101 (1980) 241.

110/T. Watanabe and H. Gerischer, J. Electroanal. Chem. 122 (1981) 73.

111/P. Krebs, K. Bukowsk, V. Giraud, and M. Heintze, Ber. Bunsenges. Phys. Chem. 86 (1982) 879.

/12/Y. Harima, H. Sato, and K. Suga, J. Phys. Chem. 93 (1989) 6418.

/13/M. Chelvayohan and C. H. B. Mee, J. Phys. C 15 (1982) 2305 .

/14/H. Raether, Surface Plasmons (Springer-Verlag, Berlin, 1988)

/15/D. M. Kolb, in Surface Polaritons, Eds. V. M. Agranovich and D. L Mills, (North-Holland, Amsterdam, 1982), p. 299; D. M. Kolb, in Trends in Interfacial Electrochemistry , Ed. A. F. Silva, (D. Reidel, Dordrecht, Holland, 1984), p. 301. /1Ei/H.-T. Chou, J. C. Villagrán, and J. C. Thompson, Phys. Rev. B XX (1991).

$/ 17 / D$. M. Riffe, G. K. Wertheim, and P. H. Citrin, Phys. Rev. Letters 64 (1990) 571. /1E/S. V. Pepper, J. Opt. Soc. Am. 60 (1970) 805.

$/ 19 /$ J. E. Sipe and G. I. Stegeman, in Surface

Polaritons, V. M. Agranovich and D.

L. Mills, Eds. (North-Holland, Amsterdam, 1982), p. 661.

120/G. Hincelin, Phys. Hev. B 24 (1981) 787. 